This item was submitted to Loughborough's Research Repository by the author.

Items in Figshare are protected by copyright, with all rights reserved, unless otherwise indicated.

\title{
Mathematical modelling of a liver hollow fibre bioreactor
}

PLEASE CITE THE PUBLISHED VERSION

https://doi.org/10.1016/j.jtbi.2019.05.008

PUBLISHER

(C) Elsevier BV

VERSION

AM (Accepted Manuscript)

PUBLISHER STATEMENT

This paper was accepted for publication in the journal Journal of Theoretical Biology and the definitive published version is available at https://doi.org/10.1016/j.jtbi.2019.05.008.

\section{LICENCE}

CC BY-NC-ND 4.0

\section{REPOSITORY RECORD}

Sorrell, lan, Rebecca Shipley, Sophie Regan, lain Gardner, Michael P. Storm, Marianne J. Ellis, John Ward, et al.. 2019. "Mathematical Modelling of a Liver Hollow Fibre Bioreactor". Loughborough University. https://hdl.handle.net/2134/37795. 
Title: Mathematical Modelling of a Liver Hollow Fibre Bioreactor

Authors: Ian Sorrell ${ }^{8}$, Rebecca J Shipley ${ }^{2}$, Sophie Regan ${ }^{6}$, lain Gardner ${ }^{3}$, Michael P Storm $^{4,10}$, Marianne Ellis ${ }^{4}$, John Ward ${ }^{5}$, Dominic Williams ${ }^{6}$, Pratibha Mistry ${ }^{7}$, José Domingo Salazar $^{9}$, Andrew Scott $^{8}$, Steven Webb ${ }^{1, *}$

\section{Affiliations:}

${ }^{1}$ Liverpool John Moores University, Department of Applied Mathematics, James Parsons Building, Byrom Street, Liverpool, L3 3AF, UK;

${ }^{2}$ Department of Mechanical Engineering, University College London, Torrington Place, London, WC1E 7JE, UK;

${ }^{3}$ Certara-Simcyp, Level 2-Acero, 1 Concourse Way, Sheffield, S1 2BJ, UK;

${ }^{4}$ Department of Chemical Engineering, Centre for Regenerative Medicine, University of Bath, Bath BA2 7AY, UK;

${ }^{5}$ Department of Mathematics, Loughborough University, Loughborough, Leicestershire, LE11 3TU;

${ }^{6}$ Drug Safety and Metabolism, IMED Biotech Unit, AstraZeneca, Cambridge Science Park, Milton Road, Cambridge, CB4 OFZ, UK;

${ }^{7}$ Syngenta Ltd., Jealott's Hill, Bracknell, Berkshire, RG42 6EY;

${ }^{8}$ Unilever, Colworth Science Park, Sharnbrook, Bedfordshire, MK44 1LQ;

${ }^{9}$ Global Medicines Development, AstraZeneca, Melbourne Science Park, Royston, SG8 6EH, UK;

${ }^{10}$ Present address: Atlas Genetics, White Horse Business Park, Wiltshire, UK.

* Correspondence author. Email: S.D.Webb@LJMU.ac.uk, Tel: +44(0) 1512312217

Short running title: Zonated hepatic bioreactor modelling

Declarations of interest: none 
Abstract: A mathematical model has been developed to assist with the development of a hollow fibre bioreactor (HFB) for hepatotoxicity testing of xenobiotics; specifically, to inform the HFB operating set-up, interpret data from HFB outputs and aid in optimizing HFB design to mimic certain hepatic physiological conditions. Additionally, the mathematical model has been used to identify the key HFB and compound parameters that will affect xenobiotic clearance. The analysis of this model has produced novel results that allow the operating set-up to be calculated, and predictions of compound clearance to be generated. The mathematical model predicts the inlet oxygen concentration and volumetric flow rate that gives a physiological oxygen gradient in the HFB to mimic a liver sinusoid. It has also been used to predict the concentration gradients and clearance of a test drug and paradigm hepatotoxin, paracetamol (APAP). The effect of altering the HFB dimensions and fibre properties on APAP clearance under the condition of a physiological oxygen gradient is analysed. These theoretical predictions can be used to design the most appropriate experimental set up and data analysis to quantitatively compare the functionality of cell types that are cultured within the HFB to those in other systems.

Key words: Hollow Fibre Bioreactor; Liver Zonation; Hepatotoxicity testing. 


\section{Introduction}

A considerable variety of screens are utilised in in vitro hepatotoxicity studies, including human and nonhuman tissue slices, primary hepatocytes (freshly isolated and cryopreserved; suspension and 2D culture), transformed liver cell lines, CYP-engineered cells, stem cell-derived hepatocytes and liver co-cultures. Drug bioactivation is thought to play a significant initiating role in the hepatotoxic mechanism of numerous drugs. Consequently a major consideration, when selecting an in vitro system for drug toxicity screening, is the normal expression and retention of xenobiotic metabolising enzymes. However, current in vitro systems show reduced liver-specific functions, in particular loss of cytochrome P450 activity (Godoy et al., 2013). It is expected that in vitro systems that mimic physiological conditions (for example in three rather than two dimensions, employing flow and nutrient gradients etc) will generate data that gives better predictions of the toxicity of xenobiotics.

Much focus and effort is being invested on the generation of in vitro systems for use in the safety assessment of new chemicals, with a growing shift in focus to human relevancy, rather than the historical approaches of using high doses in animals and extrapolating these to low exposure potential effects in humans. Many local exposure models exist, but there remains a significant need for dose-dependent systemic toxicity readouts and endpoints, such as those often seen in hepatotoxicity. Early studies using animal-derived primary cells can provide a route by which to validate these models using existing historical data, with the long term objective to use human cells to better predict in vivo exposure and thus potential toxicity in humans. Development of reliable, species-relevant and thus more predictive in vitro systems will not only produce better quality scientific data in the future but also reduce the often large numbers of animals used for these types of toxicity testing, with associated minimisation in time and cost to companies.

However, setting the operating conditions and choosing the most appropriate design to mimic physiological environments in such systems purely by experimental trial and error would be difficult, costly and time 
consuming. Also, assessing the behaviour of individual cells in systems that are by necessity spatially heterogeneous would require intensive experimental effort to accomplish. In these situations, theoretical models can provide insights, e.g. by interpolating experimental measurements to provide spatial information - thus reducing the number of measurements required-and aiding in the experimental design of these novel systems by determining theoretical relationships between the parameters and experimental observations.

Physiologically, the liver plays a key role in integrating fuel storage, endogenous metabolism, particularly lipid and glutathione metabolism, and requires significant $\mathrm{O} 2$ to perform this role (Suzuki, Shinjo, Arai, Kanai, \& Goda, 2014). This results in steep oxygen gradients across liver lobules and a high sensitivity to local oxygen concentrations. Hepatocytes make up $80-85 \%$ of the liver and are responsible for the majority of liver xenobiotic metabolism; they are arranged along short capillaries known as liver sinusoids. The function of hepatocytes is heterogeneous along the sinusoid and the oxygen gradient within the sinusoid is thought to play a key role in this heterogeneous functionality-the blood oxygen partial pressure ranges from $65 \mathrm{mmHg}$ at the sinusoid inlet to $30 \mathrm{mmHg}$ at the outlet (Jungermann \& Kietzmann, 2000). The ability to model a hepatic sinusoid in vitro, recapitulating oxygen-sensitive functionality is crucial for understanding zonal responses to hepatotoxins and hepatic disease. Hepatic hypoxia is a key feature of a number of medical conditions, and has been implicated in the development of both alcoholic and nonalcoholic fatty liver disease (Suzuki et al., 2014).

In vitro test systems that attempt to replicate this heterogeneous liver environment show improved hepatocyte functionality compared to traditional two-dimensional culture systems (Allen, Khetani, \& Bhatia, 2005). Bioreactors that use flow to induce an oxygen gradient have also shown heterogeneous distributions of cytochrome P450 enzymes-a family of metabolising enzymes frequently implicated in the formation of harmful reactive metabolites - that correlate to those seen in vivo (Allen et al., 2005). Hollow fibre bioreactor (HFB) systems incorporate physiological features such as flow and oxygen gradient required to represent a liver sinusoid in vitro and have shown promise for use as bioartificial livers (Jasmund, Langsch, Simmoteit, \& Bader, 2002; Lu et al., 2005). Extending HFB for hepatotoxicity testing is 
therefore a natural extension of this work but obviously requires validation to determine first whether the HFB can recapitulate the hepatic sinusoidal oxygen gradient and subsequent cell zonation.

Primary human hepatocytes in monolayer cultures are generally still considered the gold standard in vitro model for metabolism studies and toxicity investigations (Kyffin et al., 2018). However, there are a number of problematic issues with this system, the main one being that this classical $2 \mathrm{D} /$ monolayer cell culture does not recapitulate the zonated 3D in vivo liver micoenvironment. Much research has therefore been directed towards designing more in vivo like 3D test systems. The most commonly used liver models are liver slices (Lerche-Langrand \& Toutain, 2000), spheroids (Soldatow, LeCluyse, Griffith, \& Rusyn, 2013), scaffold and hydrogel technologies (Tibbitt \& Anseth, 2009), liver organoids (Nantasanti, de Bruin, Rothuizen, Penning, \& Schotanus, 2016) and micro-fluidic test systems (Rothbauer, Zirath, \& Ertl, 2018)-a cross comparison of the advantages and disadvantages of these different systems are reviewed in (Kyffin et al., 2018). As regards to the bioreactor system the main advantages are that: 1 ) liver cell lines (such as HepG2) cultured in this system show improved phase I metabolism compared to static 2D experiments; 2) cells on the bioreactor are polarised and form functional bile canalicular; 3 ) it is possible to achieve in vivo like liver sinusoidal zonation; 4) the cells are protected from shear stress due to being cultured on the outside of the fibre membrane (analogous to the endothelial cell lining in vivo) (Storm et al., 2016). The main limitations, however, of the bioreactor system are that it's costly and low throughput system compared to, for example, 3D spheroids or the classical 2D sandwich or monolayer cultures, plus there are well known issues regarding cell attachment on such cylindrical fibres (Storm et al., 2016).

The strategy we adopted was to use paracetamol (APAP) as a paradigm hepatotoxin to assess the phase I and II metabolic functionality of cells within the HFB for comparison with other in vitro test systems. APAP is a well understood hepatotoxin (Bessems \& Vermeulen, 2001; Hinson, Roberts, \& James, 2010) and has been used as such in many experimental studies including other bioreactors (Allen et al., 2005; Prot et al., 2011; Xia et al., 2009). It undergoes both phase I metabolism - via CYP450 enzymes to a harmful reactive metabolite (NAPQI, N-acetyl-p-benzoquinone imine) - as well as phase II metabolism. This makes APAP an ideal test compound to assess the HFB against other systems. 
In the present study a mathematical model is proposed to (a) set operating conditions for a HFB to achieve a more physiologically-relevant cellular environment and (b) quantitatively assess cellular functionality with respect to APAP metabolism. The mathematical model comprises a system of partial differential equations (PDEs) that describe the relationships between the inlet oxygen concentration, flow rate, cell density and the oxygen gradient along the HFB-this is similar to previous models of oxygen consumption in HFBs for use as bioartificial livers (Davidson, Ellis, \& Chaudhuri, 2010, 2012; Shipley et al., 2011) . By changing these parameters the system can be optimised such that the oxygen gradient along the HFB mimics that along a liver sinusoid in vivo. In addition, the metabolism of APAP within the HFB has been mathematically modelled so that we can predict how the design of the HFB-dimensions, fibre permeability and cell density-will impact on the concentration gradient of APAP along the HFB.

Mathematical models for APAP metabolism have been published previously (Diaz Ochoa et al., 2012; Pery et al., 2013; Reddyhoff, Ward, Williams, Regan, \& Webb, 2015; Reith et al., 2009; Remien, Adler, Waddoups, Box, \& Sussman, 2012; Williams et al., 2013) that use various forms of kinetics to represent the metabolism pathways - these include linear approximations and single and double substrate MichaelisMenten functions. In this paper, we use single substrate Michaelis-Menten kinetics to represent the three main pathways of APAP metabolism: sulphation, glucuronidation, and CYP activation. We predict how the design parameters and operating conditions of the HFB impact on the observed clearance rates of APAP. This is important for comparing the functionality of cells within the HFB to other in vitro test systems and for extrapolating the results to in vivo systems. It is also useful for optimizing the design of the HFB for specific experimental tasks.

In addition, an analytical approach (Shipley et al., 2011) that gives a solution applicable when the metabolism kinetics are approximately constant has been extended to give a novel analytic solution applicable when the metabolism kinetics are linear. This can then be applied to give an approximate analytic solution when the metabolism kinetics are non-linear by using a piecewise linear approximation to the metabolism function. This allows the operating conditions of the bioreactor to be determined in a more accessible form that does not require proprietary numerical software to solve PDEs. 


\section{Methods}

The current design of the bioreactor consists of a single hollow fibre contained within a glass cylinder. The fibre is a synthetic porous membrane containing a hollow central lumen and surrounded by a monolayer of confluent cells. The region outside of this, contained within the glass cylinder, is referred to as the extracapillary space (ECS). Let $z$ be the axial direction down the lumen centreline, with $z=0$ at the inlet and $z=\mathrm{L}$ at the outlet. The radius of the lumen is denoted by $d$, the depth of the membrane by $s$ and the depth of the ECS is by I. Figure 1 shows a schematic of the bioreactor; we have assumed radial symmetry so that there is no aximuthal variation in the geometry of the HFB, and denote the radial coordinate by $r$, with $r=0$ corresponding to the lumen centreline. The dimensions of the bioreactor for initial testing (and typical of experimental values used) will be $L=0.1 \mathrm{~m}, d=300 \mu \mathrm{m}, s=200 \mu \mathrm{m}, I=100 \mu \mathrm{m}$, with these values subject to change depending on design criteria and mathematical predictions. The dimensions of the bioreactor were selected so that, with the given cellular $\mathrm{O}_{2}$ consumption rates and pump flow ranges, the oxygen gradient along the length of the bioreactor recapitulated the $65 \mathrm{mmHg}$ (at the inlet) to $30 \mathrm{mmHg}$ (at the outlet) gradient observed in the real in vivo liver sinusoid. We assume a cell layer surface density of $\rho=$ $1.32 \times 10^{9}$ cells per $\mathrm{m}^{2}$ based on assuming the area of membrane occupied by a hepatocyte is a regular hexagon with maximal diameter $34.2 \times 10^{-6} \mathrm{~m}$ (Katayama, Tateno, Asahara, \& Yoshizato, 2001); we explore small variations in this density to investigate the uncertainty in the cell seeding process and the shape of individual hepatocytes within the monolayer. Parameter values for both oxygen and APAP transport are summarised in Table 1. The membrane offers resistance to diffusion of compounds through it compared to free space, dependent on the pore size and tortuosity of the membrane material; we capture this through a membrane hindrance factor, $h=10$, such that $D_{m}=D_{l} / h$ for both oxygen and APAP (and subsequently explore the sensitivity of model predictions to variations in $h$ ). 


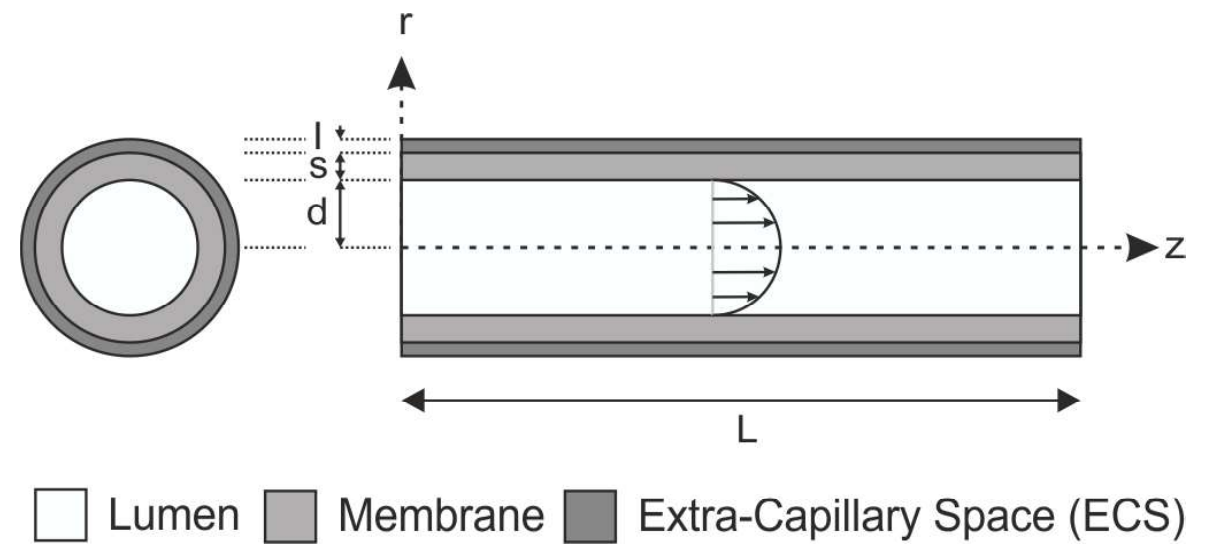

Figure 1: Schematic of a hollow fibre bioreactor. The fibre consists of a single fibre shown in radial (left) and axial (right) cross section. Cells are seeded in a confluent monolayer on the outer membrane surface $(r=d+s)$. Culture medium is pumped into the lumen inlet at $z=0$, and exists via the lumen exit at $x=L$. A typical fluid velocity profile is shown on the right.

Table 1: Parameters for Oxygen and APAP. ${ }^{\mathrm{a}}$ To convert $V_{\max }$ units from source, a rat liver volume fraction of 0.037 (Pery et al., 2013) has been used.

\begin{tabular}{lcc} 
Parameter & Value & Source \\
\hline Oxygen & & \\
\hline$D_{l}$ and $D_{e}$ & $3 \times 10^{-9} \mathrm{~m}^{2} \mathrm{~s}^{-1}$ & (Shipley et al., 2011) \\
$D_{m}=D_{l} / h$ & $3 \times 10^{-10} \mathrm{~m}^{2} \mathrm{~s}^{-1}$ & (Shipley et al., 2011) \\
Rat $I n$ Vivo Hepatocytes: & & \\
$V_{\max }$ & $1.76 \times 10^{-3} \mathrm{~mol} \mathrm{~m}^{-3} \mathrm{~s}^{-1}$ & (Shipley et al., 2011) \\
$K_{m}$ & $6.24 \times 10^{-3} \mathrm{~mol} \mathrm{~m}^{-3}$ & (Shipley et al., 2011) \\
& & \\
Paracetamol (APAP) & & (Ribeiro et al., 2012) \\
\hline$D_{l}$ and $D_{e}$ & $0.65 \times 10^{-9} \mathrm{~m}^{2} \mathrm{~s}^{-1}$ & - \\
$D_{m}=D_{l} / h$ & $0.65 \times 10^{-10} \mathrm{~m}^{2} \mathrm{~s}^{-1}$ & \\
Primary Rat Hepatocytes: & & (Pery et al., 2013) \\
Glucuronidation $V_{\max }$ & $2.4 \times 10^{-3} \mathrm{~mol} \mathrm{~m}^{-3} \mathrm{~s}^{-1}$ & (Pery et al., 2013) \\
Glucuronidation $K_{m}$ & $0.915 \mathrm{~mol} \mathrm{~m}^{-3}$ &
\end{tabular}

Fluid (culture medium) enters the system through the lumen inlet $(z=0)$ at a designated constant flow rate and exits via the lumen outlet $(z=L)$; there is no flow of fluid in the membrane or ECS spaces. Although other compounds are contained within the media, we only consider the transport of oxygen and the hepatotoxin (here APAP). In the lumen, compounds are transported by both advection and diffusion, whereas diffusion is the only transport process in both the membrane and the outer cylinder. We also assume that cells form a confluent monolayer that is fixed in time. This is a reasonable approximation as on the timescale we are considering for transport processes, cell proliferation and death are quasi-steady. The 
cell monolayer is thin compared to the lumen radius and membrane, ECS depths (10 $\mu \mathrm{m}$ compared to 100 $300 \mu \mathrm{m})$, therefore we assume that the compound concentration in the cell layer is constant in the radial direction normal to the membrane surface. Indeed, in the experimental set-up (see (Storm et al., 2016)), the cells are observed to flatten to form a single cell monolayer on the outside of the fibre therefore such a thin boundary layer assumption seems valid. There may be cases (e.g. different cell types) where the cells may grow and form multiple layer cell structures on the outside of the fibre. In which case, multiple layers of cells can be included in the model as multiple radial compartments within the PDE framework and it is possible that an analytic solution could be computed for this case using similar methods as to those used in this paper, but this is beyond the scope or focus of this current work. Under the thin boundary layer assumption the metabolism of compound by the cells can be modelled as a jump condition in the flux at the membrane/ECS boundary (this assumption was verified numerically compared to one where the cell layer thickness was explicitly accounted for, with details provided in the Supplementary Material).

We also assume that the intrinsic clearance rate of a compound is independent of the oxygen level. There is experimental evidence that the rates of the main APAP metabolism pathways of sulphation and glucuronidation are independent of the position of the cell along the sinusoid, which implies they are also independent of the oxygen concentration (Anundi, Lahteenmaki, Rundgren, Moldeus, \& Lindros, 1993). This is not the case for the turnover of APAP by CYP activation - which is heterogenous along the sinusoid (Allen et al., 2005) - however, this is a relatively minor pathway for considerations of APAP elimination, with around 5\% of APAP being metabolised through this pathway (Remien et al., 2012; Siegers, Rozman, \& Klaassen, 1983).

Fluid flow in the lumen is within a low reduced Reynolds number regime, and we follow the approach of (Shipley et al., 2011) and describe it using Poiseuille flow so that the fluid velocity, $u=2 U\left(1-r^{2} / d^{2}\right) \boldsymbol{e}_{z}$, where $U$ is the mean velocity $\left(\mathrm{ms}^{-1}\right)$ and $\boldsymbol{e}_{z}$ is the unit vector in the z-direction. Both oxygen and APAP are modelled by the same set of conservation equations differing only in values of the respective diffusion coefficients and the Michaelis-Menten constants that parameterise cellular metabolism, as given in Table 1. We denote the concentration of chemical (here either oxygen or APAP) by $c\left(\mathrm{~mol} \mathrm{~m}^{-3}\right)$ with subscripts $l, m$, 
and $e$ denoting the values in the lumen, membrane and extra-capillary space. The steady state conservation equations in each region are:

$$
\begin{aligned}
& \nabla \cdot\left(c_{l} \boldsymbol{u}\right)=D_{l} \nabla^{2} c_{l} \text { in the lumen, } \\
& 0=D_{m} \nabla^{2} c_{m} \text { in the membrane, } \\
& 0=D_{e} \nabla^{2} c_{e} \text { in the ECS, }
\end{aligned}
$$

where $D_{l} D_{m}$, and $D_{e}$ are the diffusion coefficients in the lumen, membrane and ECS respectively-all assumed constant, with units $\mathrm{m}^{2} \mathrm{~s}^{-1}$. It is necessary to specify boundary conditions on the external boundaries of the bioreactor and between its internal regions. Continuity of concentration and flux is imposed upon the boundaries between the lumen and membrane, given by

$$
c_{l}=c_{m} \text { and } D_{l} \nabla c_{l} \cdot \boldsymbol{n}=D_{m} \nabla c_{m} \cdot \boldsymbol{n} \text { on the lumen/membrane boundary, } r=d,
$$

where $\boldsymbol{n}$ is the outward pointing unit vector normal to the relevant surface. Continuity of concentration is also imposed upon the boundary between the membrane and ECS and the metabolism of compound by the cells is represented by a jump condition in the flux at this boundary. This gives

$$
\begin{gathered}
c_{m}=c_{e} \text { and } \frac{1}{\varphi}\left(D_{m} \nabla c_{m} \cdot \boldsymbol{n}-D_{e} \nabla c_{e} \cdot \boldsymbol{n}\right)=R\left(c_{m}\right) \\
\text { on the membrane/ECS boundary, } r=d+s,
\end{gathered}
$$

where $\varphi=\rho v_{\text {hep }}$ with the volume of a hepatocyte $v_{\text {hep }}=6.98 \times 10^{-15} \mathrm{~m}^{3}$ (Plettenberg, Weiss, Lemor, \& Wehner, 2008) and $R(c)\left(\mathrm{mol} \mathrm{m}^{-3} \mathrm{~s}^{-1}\right)$ is a function representing cellular metabolism - this will typically be a Michaelis-Menten function. A zero flux condition is imposed on the outer ECS boundary, that is, $D_{m} \nabla c_{m}$. $\boldsymbol{n}=0$ on $r=d+s+l$. This represents no transport out of the glass cylinder. At the lumen inlet $(z=0)$ the concentration is given by $c_{l}=c_{i n}$, where $c_{\text {in }}$ is a fixed concentration prescribed experimentally to give appropriate functionality within the bioreactor. Zero diffusive flux is also imposed on the ends of the bioreactor at $z=0, L$ within the membrane and ECS domains (which are closed there), and at $z=L$ within the lumen (which imposes a minimal restriction on the lumen concentration field). The steady state of this PDE system of equations (1)-(5) can be solved numerically-we have done this using the COMSOL Multiphysics 
software package-but for a linear cellular metabolism function an explicit analytic solution has also been derived. Furthermore, this solution can be applied to an arbitrary metabolism function provided it is differentiable. The full details of the derivation of the analytic solutions are in the Supplementary Materials with a brief summary below. The COMSOL simulations were carried out with a mesh containing 698 elements (the mesh was refined up to 1964 elements with no change in the concentration predictions to 2 significant figures).

To make progress analytically the system of equations given by (1)-(5) is reduced asymptotically by exploiting the small aspect ratio of the fibre defined by $\varepsilon=\frac{d}{L} \approx 3 \times 10^{-3} \ll 1$, and integrating the resulting equation set. Note that although the lumen radius $d$ and length $L$ can be varied in the design of the HFB, $\varepsilon \ll 1$ will always hold. For the simplification to be valid a second condition must be met; the reduced Péclet number $P_{e}=\frac{U d^{2}}{L D_{l}} \approx 0.1$ should satisfy $P_{e}>o(\varepsilon)$. This condition is equivalent to ignoring diffusion in the axial z-direction due to a combination of the dominance of advection in the lumen and the small aspect ratio. This simplification process and the criticality of the reduced Péclet number have been previously used and discussed in (Shipley et al., 2011; Shipley, Waters, \& Ellis, 2010).

We have derived explicit analytic steady-state solutions to the system of equation (1)-(5) in the cases where the metabolism function $R(\cdot)$ is linear, that is $R\left(c_{e}\right)=A_{R} c_{e}+B_{R}$, for constants $A_{R}$ and $B_{R}$, and also where the cellular metabolism is represented by a Michaelis-Menten function of the form $R\left(c_{e}\right)=V_{\max } c_{e} /\left(K_{m}+\right.$ $c_{e}$ ), where $V_{\max }$ is the maximum velocity and $K_{m}$ is the Michaelis-Menten constant, given by the concentration at which the reaction rate is half maximal. The metabolism functions of oxygen and APAP are represented here by Michaelis-Menten functions in common with other work for oxygen (Davidson et al., 2010; Hay, Veitch, \& Gaylor, 2001; Shipley et al., 2011) and for APAP (Pery et al., 2013; Reith et al., 2009). For Michaelis-Menten metabolism functions, the analytic solutions can be used to approximate compound gradients in three different ways. The most suitable method depends on the parameter $K_{m}$ and the concentration of compound within the bioreactor at the cells: 
1. If $c_{e} \gg K_{m}$, then the zero order approximation $R\left(c_{e}\right) \approx V_{\max }$ is applicable. The can then be used to calculate the compound gradient by letting $A_{R}=0, B_{R}=V_{\max }$ (this is analogous to the analytic solutions for a zero order approximation previously derived by the same method (Shipley et al., 2011)).

2. If $c_{e} \ll K_{m}$ then the first order approximation $R\left(c_{e}\right) \approx \frac{V_{\max } c_{e}}{K_{m}}$ is applicable. In this case, the solution can be used to calculate the gradient by letting $A_{R}=\frac{V_{\max }}{K_{m}}, B_{R}=0$.

3. If neither the first order nor zero order approximations are applicable, or greater accuracy is required, a piecewise linear approximation scheme can be implemented to compute the compound gradients. This procedure can be applied to an arbitrary metabolism function as long as it is differentiable and full details of method are given in the Supplementary Materials. A version of this is also implemented in the Excel spreadsheet in the Supplementary Materials, which could be applied by an end-user, for example, to characterise the concentration fields in their bioreactor.

The average clearance per cell is used as a measure of observable cell functionality within the bioreactor that could be measured experimentally from the outlet concentration of compound leaving the bioreactor. If $Q_{\text {in }}$ is the molar amount of compound entering the HFB inlet per second, $Q_{\text {out }}$ is the quantity of compound leaving the HFB outlet per second and $T_{\text {cells }}$ is the total number of cells in the bioreactor, then the average clearance per cell is calculated by $\left(Q_{\text {in }}-Q_{\text {out }}\right) / T_{\text {cells }}$.

\section{Results}

Simulations for predicted concentrations of oxygen and APAP within the bioreactor are shown in Figure 2 . The input oxygen concentration and fluid flow rate have been set to reproduce physiological oxygen gradients within the bioreactor at the cell density simulated. The predicted clearance of APAP under these conditions is also shown. The parameters that determine the rate of clearance - that is the parameters for sulphation, glucuronidation-are based on in vivo estimates (Pery et al., 2013). The cells are predicted to clear over $50 \%$ of the inlet concentration of APAP which is similar to what has been seen in liver perfusion studies of isolated whole rat livers (Pang, Barker, Simard, Schwab, \& Goresky, 1995), where clearances of $50 \%$ have been observed. Hepatocytes in vitro typically have reduced functionality compared to 
hepatocytes in vivo. Figure 3 shows predicted APAP clearance rates in an HFB populated by cells that have elimination rates calculated from literature in vitro studies carried out in conventional 2D well-plate systems (rat and cryopreserved human hepatocytes in suspension (Naritomi, Terashita, Kagayama, \& Sugiyama, 2003)), as well as a microfluidic in vitro platform (Prot et al., 2011) and in vivo with clearance rates calculated from relevant PBPK models (Pery et al., 2013; Watari, Iwai, \& Kaneniwa, 1983). The relationship between elimination rates of APAP by the cells and the average clearances within the HFB are shown in Figure 3B. Expressed on a log-log scale, this relationship is approximately linear with slope decreasing slightly with increasing intrinsic clearance. The corresponding radial APAP concentration changes at the outlet of the HFB are shown in Figure 3A. This shows a marked difference in concentration between the lumen $\left(r<3 \times 10^{-4} \mathrm{~m}\right)$ and the cell layer $\left(r=5 \times 10^{-4} \mathrm{~m}\right)$ for cells with a high elimination rates for APAP. This means that differences between cell types and in vitro test systems can be clearly observed by taking account of the concentration of the remaining compound in the media at the outlet flow of the HFB (and thereby determining average HFB clearance) as well as the radial compound gradient within the bioreactor. These results also highlight the magnitude of the differences between cell functionalities in some current in vitro systems compared to that in vivo, and the gap that we are attempting to fill with this new optimised HFB system. The goal is to achieve average clearances from cells in the HFB at levels comparable to that predicted for in vivo and the aim of this mathematical modelling is to optimise the HFB to recapitulate the best physiologically representative conditions in the HFB to achieve this. 

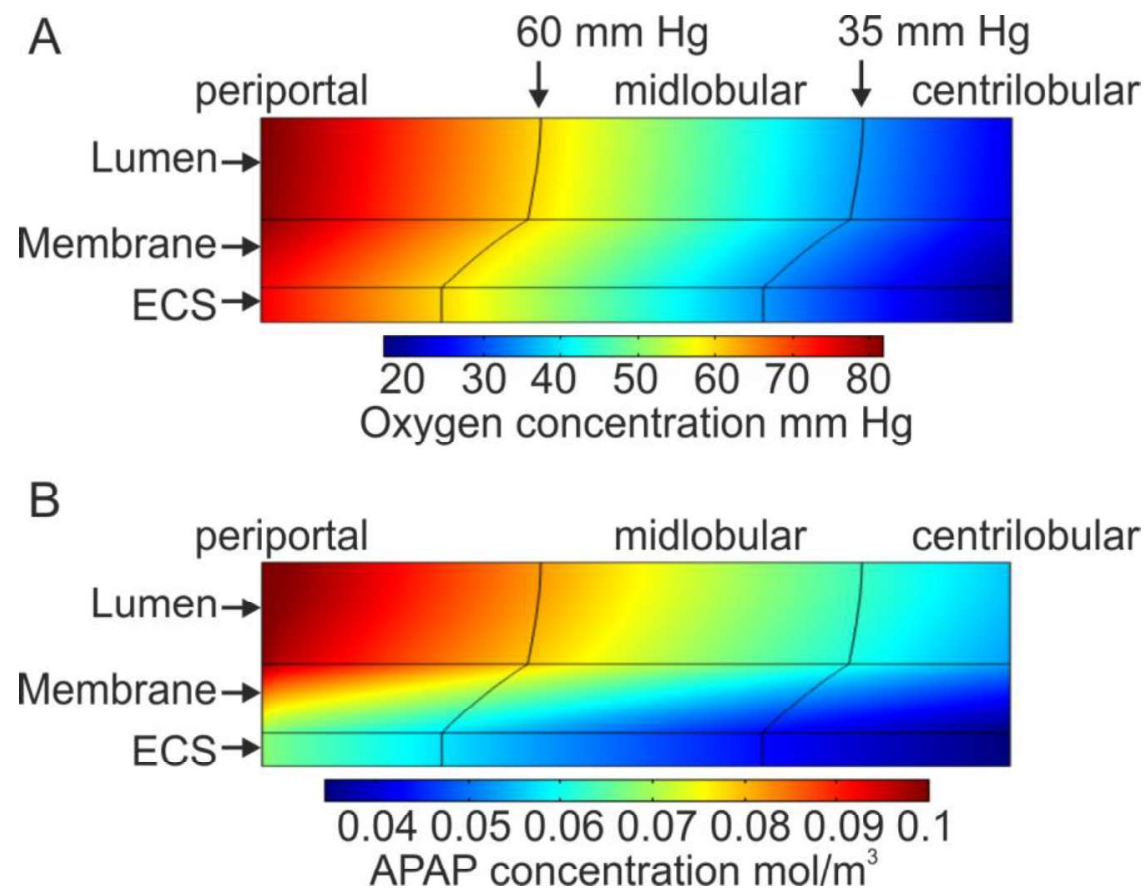

Figure 2: Numerical simulation of concentration gradients of oxygen and APAP within the bioreactor. (A) Oxygen and (B) APAP within a hollow fibre bioreactor seeded with primary rat hepatocytes with zonal regions indicated. The mean flow rate along the lumen was $U=0.0005 \mathrm{~ms}^{-1}$. The inlet concentrations of oxygen and APAP were $67 \mathrm{mmHg}$ and $0.1 \mathrm{~mol} / \mathrm{m}^{3}$ respectively. Other bioreactor parameters were $\mathrm{L}=0.1 \mathrm{~m}$, $\mathrm{d}=3 \times 10^{-4} \mathrm{~m}, \mathrm{~s}=2 \times 10^{-4} \mathrm{~m}, \mathrm{l}=1 \times 10^{-4} \mathrm{~m}$.

A

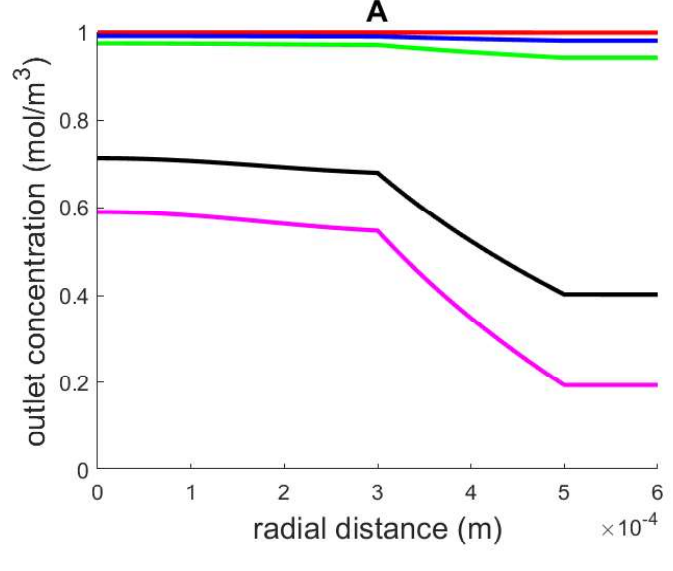

B

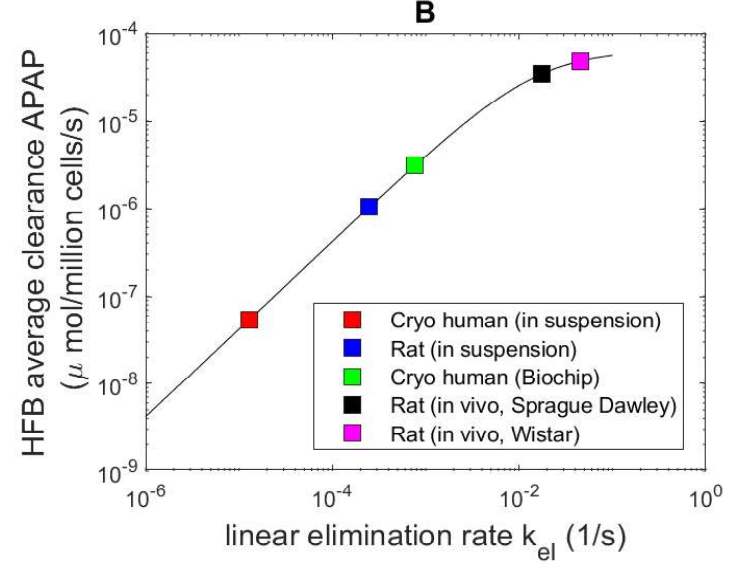

Figure 3: Predictions of radial concentrations of APAP in the HFB outlet and clearance rates determined in different in vitro and in vivo models. (A) Radial concentrations of APAP at the HFB outlet and (B) average clearance rates with elimination rates determined from different hepatocyte systems - rat in vivo (Pery et al., 2013; Watari et al., 1983), rat and cryopreserved human cells in suspension (Naritomi et al., 2003) and cryopreserved human hepatocytes in a microfluidic in vitro platform . In order to incorporate these experimental elimination rates $\left(k_{e l}\right.$; measured with units per second) in the model, the APAP metabolism term is reduced to linear/first order approximation form, $V=k_{e l} c \varphi$, with $\varphi=\rho v_{h e p}$ and $k_{e l}=4.58 \times 10^{-2} s^{-1}$ (Rat Wistar in vivo (Watari et al., 1983)); $k_{e l}=1.732 \times 10^{-2} s^{-1}$ (Rat Sprague Dawley in vivo (Pery et al., 2013)); $k_{e l}=7.707 \times 10^{-4} s^{-1}$ (Cryo human Biochip (Prot et al., 2014)); $k_{e l}=2.483 \times 10^{-4} s^{-1}$ (Rat suspension (Naritomi et al., 2003)); $k_{e l}=1.2916 \times 10^{-5} s^{-1}$ (Cryo human 
suspension (Naritomi et al., 2003)). APAP dose is $1 \mathrm{~mol} / \mathrm{m}^{3}$ and the other parameters are as given in Table 1.

The different methods for computing steady state concentrations are compared in Figure 4. The analytic solution with a zero order approximation (Figure 4A) and first order approximation (Figure 4B) agree well with the numerical solution of the full system of PDEs computed using COMSOL. The analytic solutions do predict a lower concentration within the bioreactor but this is to be expected as the zero order and first order approximations both give a higher clearance rate than the Michaelis-Menten function from which they are derived. There is extremely good agreement between the solution computed from solving the full system of PDEs numerically and the one computed by the piecewise scheme; percentage errors are less than $0.25 \%$ for a scheme using ten linearization steps-details of the percentage error calculation is contained in Supplementary Material.
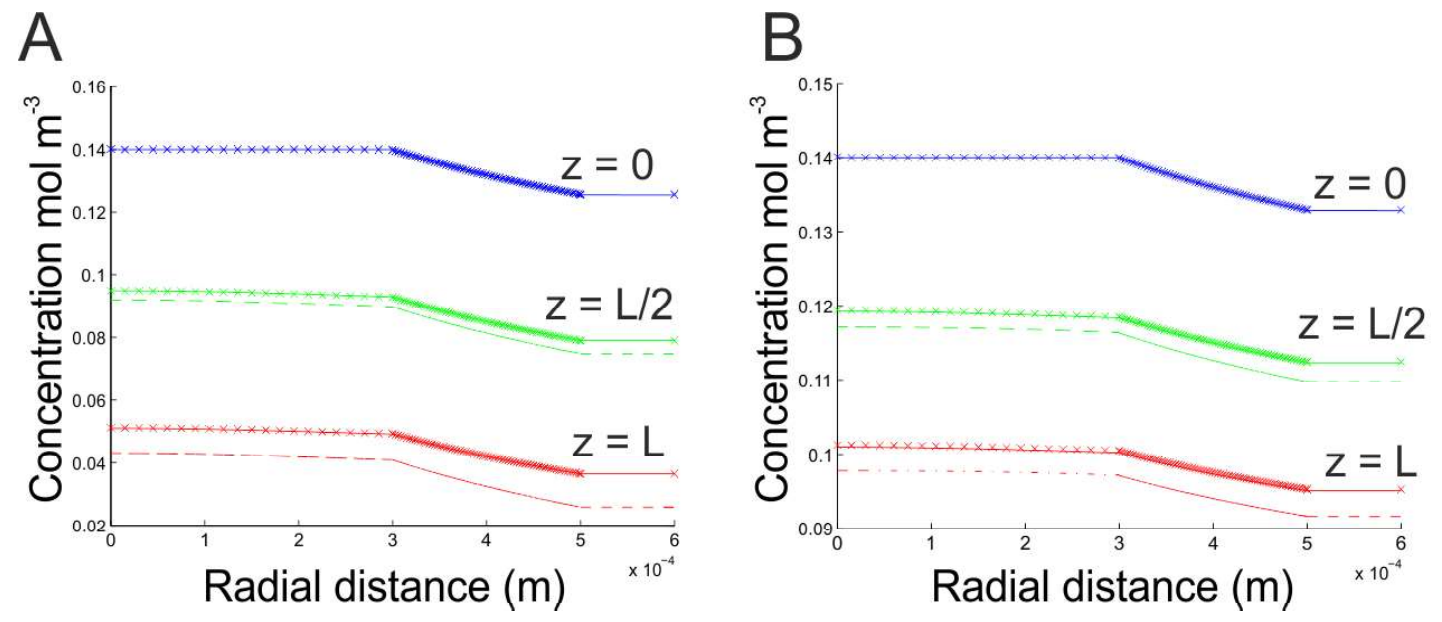

Figure 4: Comparison of numerical and analytic approaches. Concentration of compound at inlet $(z=0$, blue), midpoint $(z=L / 2$, green) and outlet $(z=L$, red) of bioreactor with cellular consumption governed by a Michaelis-Menten reaction with (A) low Michaelis constant $K_{m}=6.24 \times 10^{-3} \mathrm{~mol} / \mathrm{m}^{3}$ and (B) high Michaelis constant $K_{m}=1 \mathrm{~mol} / \mathrm{m}^{3}$. Solid lines are computed numerically using COMSOL, dashed lines are the appropriate analytic approximation-(A) zero order and (B) first order-and crosses $(x)$ are computed using the piecewise linear scheme. In (A) $V_{\max }=1.76 \times 10^{-3} \mathrm{~mol} \mathrm{~m}^{-3} \mathrm{~s}^{-1}$ and in (B) $V_{\max }=7 \times 10^{-3} \mathrm{~mol} \mathrm{~m}^{-3} \mathrm{~s}^{-1}$. All other parameters identical between $(A)$ and $(B)$ and given in Table 1.

We have carried out analysis to understand how sensitive clearance rates within the HFB are to variations in HFB design and operation parameters (Figure 5). The relationship between volumetric flow rate and the radius of the lumen, membrane hindrance factor and depth that results in a fixed oxygen gradient is shown 
in Figure 5. To mimic physiological conditions the oxygen partial pressure at the cell layer at the inlet end of the HFB is set to be $65 \mathrm{mmHg}$, whilst at the outlet end it is set to be $30 \mathrm{mmHg}$ by adjusting the flow rate of media down the lumen and the inlet oxygen concentration. We have also looked at how sensitive the clearance rates are to changes to the volumetric flow rate that result in an oxygen outlet concentration at the cells of up to plus or minus $10 \%$ of the base level. Within this range the clearance of APAP has been plotted (Figure 5). The relationship between the length of the HFB and the volumetric flow rate required to maintain a fixed oxygen gradient has been calculated analytically. In the analytic solutions, length $L$ and flow rate $U$ only appear in the Péclet number $P_{e}=\frac{U d^{2}}{L D_{l}}$. Therefore, increasing both the average velocity in the lumen and the HFB length by the same proportional amount will produce an identical concentration gradient. The three colour plots, Figures $5 \mathrm{~A}, \mathrm{C}$ and $\mathrm{E}$, show that altering the volumetric flow rate to give an oxygen concentration up to plus or minus $10 \%$ of the base level has little effect on the predicted average clearances. 
A

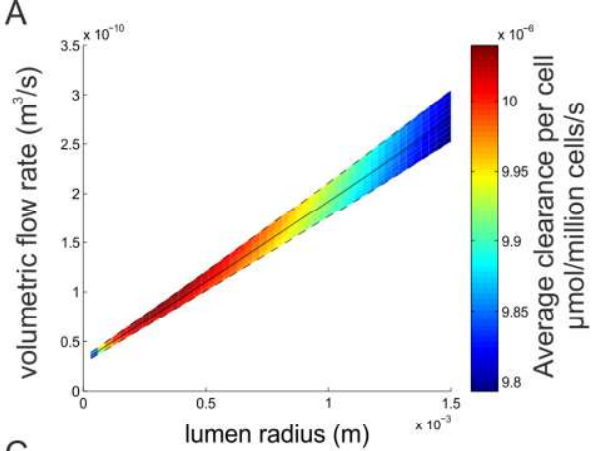

C

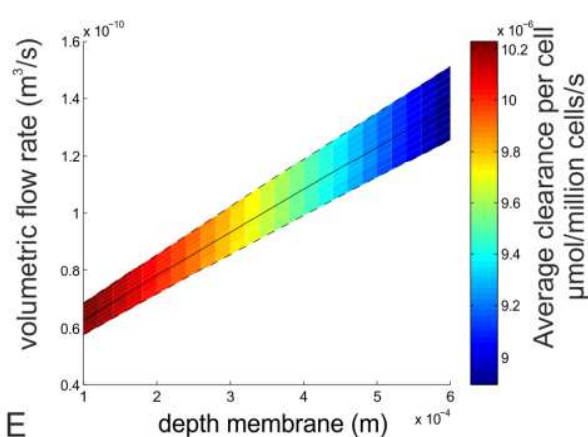

E

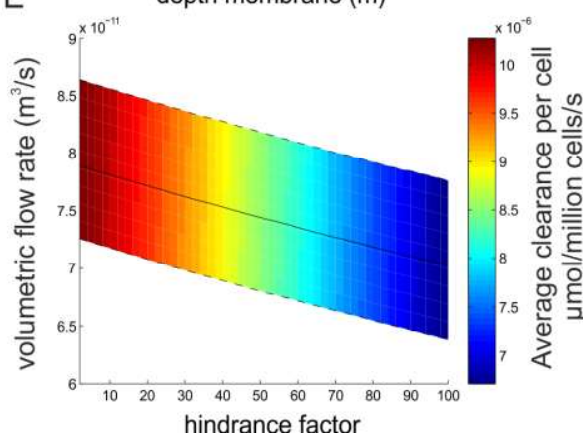

B
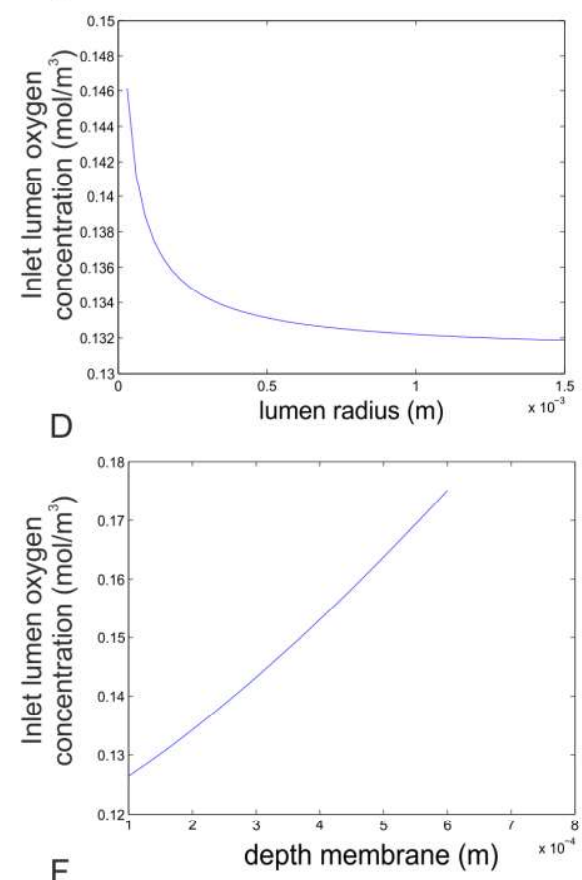

F

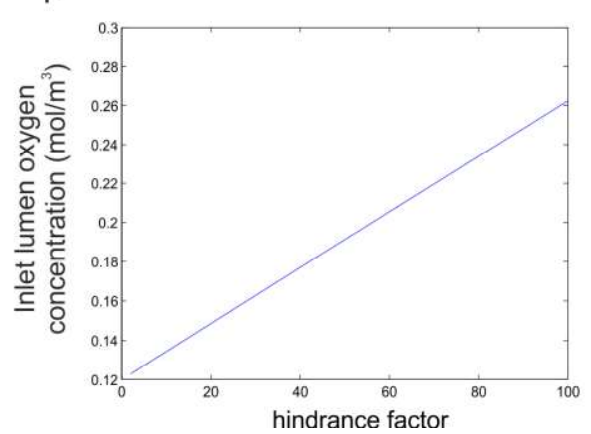

Figure 5: Sensitivity analysis of the HFB model. Relationship between either volumetric flow rate $(A, C, E)$ or inlet lumen oxygen concentration $(B, D, F)$ and lumen radius $(A, B)$, membrane depth $(C, D)$ and membrane hindrance factor $(E, F)$, shown together with average clearance of APAP $(A, C, E)$. Solid black line $(A, C, E)$ indicates where the oxygen concentration at the cell monolayer is the base level of $0.12 \mathrm{~mol} / \mathrm{m}^{3}$ at the inlet end of the bioreactor and $0.054 \mathrm{~mol} / \mathrm{m}^{3}$ at the outlet end. The dashed black lines indicate where the oxygen levels are $10 \%$ above or below the base level. The colour map shows the average clearance of APAP. The inlet concentration of APAP is $1 \mathrm{~mol} / \mathrm{m}^{3}$. Other parameters are as given in Table 1 .

If the HFB lumen radius is varied (and other geometry/ operating conditions chosen to give the same oxygen gradient at the cell layer), the average clearance rates will remain very similar (Figure $5 \mathrm{~A}$ ), independent of the APAP dose (also see Figure $3 \mathrm{~A}$ in supplementary materials). This suggests that the radius of the lumen can be determined by other considerations such as the number of cells - a larger radius will result in a larger outer membrane surface area and require more cells to form a confluent monolayerthat will be influenced by the seeding method, cell growth rates and the sensitivity of the measurements of the system outputs. In Figure 5B, the key result is that a lower lumen inlet $\mathrm{O} 2$ concentration is needed to 
maintain $65 \mathrm{mmHg}$ at the inlet part of the cell layer when the radius of the lumen is larger. At smaller lumen radii, there is a lower total amount of oxygen available to the cells, so the lumen input concentration needs to be increased to compensate. At higher lumen radii, depletion doesn't occur so the required lumen oxygen concentration remains relatively constant.

There is a more significant difference in average clearance rates for HFBs that differ in membrane depth (Figure $5 \mathrm{C}$ ) and this especially true at low doses of APAP where the concentration gradient in the bioreactor will be large relative to the inlet concentration (also see Figure 3 B in supplementary materials). This is due to the relative rates of diffusive and advective transport in the lumen and membrane. This can be seen in Figure $3 \mathrm{~A}$ where concentration decreases rapidly radially in the membrane due to the slower rate of diffusion compared to the total transport in the lumen. To achieve the same oxygen gradient for different membrane depths requires adjustments in volumetric flow rate and inlet oxygen concentration as shown in Figure $5 \mathrm{C}$ and $\mathrm{D}$ respectively. To adjust the input concentration of an arbitrary compound to achieve a particular concentration at the cells at the inlet end would require detailed knowledge of the compound consumption/turnover rates of the cells within the HFB. If the volumetric flow rate is fixed by the oxygen concentration it would then not be possible to set the gradient of the second compound arbitrarily and this must be factored into the experimental design using the mathematical model predictions for guidance.

Altering the hindrance factor, $\mathrm{h}$, that characterises membrane diffusion also has an impact on average clearance, with the effect more pronounced for low doses of APAP (Figure $5 \mathrm{E}$ and supplementary Figure 3C). This is important if the hindrance factor increases during the operation of the bioreactor due to chemical and protein fouling. The oxygen gradient can be maintained by adjusting the flow rate but this will impact upon the clearance of APAP especially at low doses. For Figures $5 E$ and F, hindrance factor doesn't have such a big effect because the rate of diffusion across the membrane isn't strongly limiting for the equilibrium. That is, it might take longer to reach equilibrium with a higher hindrance factor but the oxygen concentration at the cell layer is mainly determined by the amount of oxygen coming in, at the lumen input, and the number of cells consuming it. It is important to note that varying the membrane depth and 
the lumen radius change the number of cells so they will both have a bigger impact on the oxygen concentrations and subsequently how much this needs to be compensated for by the changing flow rate and inlet oxygen concentration. The equilibrium concentrations are less sensitive to the rate at which oxygen is transported across the fibre membrane, but the time to reach the equilibrium will clearly be impacted.

At low doses the average clearance of APAP is also significantly affected by the density of cells (Figure 1 Supplementary Materials) even when the volumetric flow rate through the HFB is being controlled to ensure the same oxygen concentration. An increase in the cell density (and therefore cell metabolism) must be compensated by an increase in flow rate to maintain the oxygen gradient. However, due to the differences in diffusivity between APAP and oxygen the average clearance per cell of APAP will decrease.

To consider bioreactor operation for compounds other than APAP, we have carried out simulations where the diffusivity of our test compound is varied (Figure 2 Supplementary Materials). A proportional change in diffusivity can be considered as the reciprocal of the same proportional change in the Stokes radius of the compound. Our simulations show that compounds with a similar rate of intrinsic clearance to APAP but a lower diffusion coefficient will have a lower total clearance in a HFB than APAP if they are administered at the same dose. This difference is not greatly affected by the membrane hindrance factor. However, lowclearance compounds-compounds with a long in vivo half-life-have a total HFB clearance that is less dependent on the diffusion coefficient. Two low clearance compounds with a similar intrinsic clearance rate will have similar total HFB clearances even if there is a marked difference in their diffusion coefficients. This is shown in the simulations of a compound with varying diffusion coefficient and a metabolism $V_{\max }$ that is $10 \%$ that of APAP.

\section{Discussion}

Currently hepatotoxicity screens that use two dimensional cultures or suspensions of primary hepatocytes and hepatic cell lines show reduced metabolic functionality compared to liver cells in vivo-for example primary hepatocytes rapidly lose CYP450 after isolation (Boess et al., 2003; Michalopoulos, Sattler, \& Pitot, 
1976). Three dimensional systems incorporating more realistic physiology have shown increased expression of these enzymes-such as the CYP450 family of enzymes (Allen et al., 2005). However, it is inherently more complex to determine the metabolic activity in three dimensional systems and there is no standard for comparing the metabolic functionality of cells of different types, used in different environments. Measuring enzyme proteins and mRNA is invasive-which is not ideal for a low-throughput device such as a $\mathrm{HFB}-$ and does not necessarily translate into functionality. Determining the rate of cellular compound turnover in a 3D system with flow is complicated by the cells not all being subject to the same external concentration-rates of metabolism, toxicity and often transport are typically concentration dependent. The analytic solutions presented here allow for the computation of chemical and compound gradients within the bioreactor using only measurements of inlet and outlet concentrations. These solutions have the advantage of being suitable for spreadsheets and purpose-built applications without the need for proprietary software. This will allow users of the HFB to access the modelling to calculate the operating conditions for their particular cell type, set up and experimental requirements. Principally this is through the calculation of the inlet oxygen concentration and flow rate required to achieve a fixed oxygen gradient along the HFB in the cell monolayer. It will also allow for the calculation of spatial cell metabolism rateseither nutrient metabolism or xenobiotic clearance-from samples of the output media alone. In addition the mathematical model could interpret changes in oxygen consumption as an early indicator of toxicity within the HFB. This is through the use of oxygen probe data from the output media to determine the key time points when toxic events are beginning to impact upon cell function. The mathematical analysis also shows that the concentration within the ECS is constant radially and will be approximately the same as the unbound concentration in the cells at any given point along the length of the bioreactor. Measuring the concentration in the ECS at the inlet and outlet ends of the HFB will give a more relevant measurement for calculating the metabolism rate of the cells than the lumen concentration. This may influence the design of the bioreactor.

Simulations of a HFB containing a monolayer of cells that have APAP elimination rates calculated from hepatocytes under different conditions-in vivo, suspension and microfluidic-show considerable differences in the total clearance within the HFB. This means that any improvement in the functionality of 
cells over those in $2 \mathrm{D}$ cultures should produce a measurable change in the total clearance in the HFB for our APAP test compound. This will allow us to quantitatively compare the HFB to 2D cultures and other systems, and such in vitro to in vivo extrapolations for human primary cells and cell lines will be a focus of our future work.

Analysing how the parameters of the HFB model change APAP clearance under the condition of maintaining the same oxygen gradient has revealed some important insights into the design of the HFB. First of all, HFBs of different lengths but with the same oxygen gradient will have identical APAP gradients. This means the length of the HFB can be chosen on other considerations for example cell number or convenience of production. Secondly, altering the radius of the lumen does not greatly change the predicted clearance of APAP. Again this is another parameter that can be chosen without considering the effect on compound clearance. Thirdly, compound clearance is highly sensitive to the membrane depth and diffusion hindrance factor. These are key parameters for the HFB as changes in the hindrance factor of the membrane may result from protein and compound fouling and there may be variability in pore size and tortuosity between batches of fibres. Our results show a HFB with a thin membrane will have higher APAP clearance than a HFB with a thicker membrane, under the condition that the HFBs are set up to have the same oxygen concentration gradient at the cells and the same input APAP concentration. How this relationship alters with compounds of different sizes and intrinsic clearances will have an impact on the development of the membrane materials and production method.

Overall, in better recapitulating the graded hepatic sinusoid oxygen microenvironment, HFB have the potential to provide more efficient, sensitive and specific tests of liver function and potential toxicity. The mathematical modelling of the HFB presented here will speed up bioreactor development, aid in experimental design and operation, allow for quantitative assessment between systems and give improved in vitro to in vivo extrapolations. We have identified the key parameters in the design of the HFB that impact on xenobiotic clearance-membrane depth, hindrance factor and cell density-and shown that these factors are only important if there is a significant xenobiotic concentration gradient in the HFB through either a low dose or low clearance rate. The parameters identified as not impacting significantly on 
xenobiotic clearance are lumen radius and length. We have also produced novel analytic results that allow

the mathematical model to be employed by non-specialists without the need for proprietary software.

Acknowledgements: The work was supported by the National Centre for the Replacement, Refinement and Reduction of Animals in Research (NC3Rs) CRACK-IT fund, Challenge 5: IVIVE. We would like to thank Cathy Vickers (N3CRs) who provided invaluable support and guidance to the IVIVE team as well as expertise and insight during team discussions.

\section{References}

Allen, J. W., Khetani, S. R., \& Bhatia, S. N. (2005). In vitro zonation and toxicity in a hepatocyte bioreactor. Toxicological Sciences, 84(1), 110-119. doi:10.1093/toxsci.kfi052

Anundi, I., Lahteenmaki, T., Rundgren, M., Moldeus, P., \& Lindros, K. O. (1993). Zonation of acetaminophen metabolism and cytochrome-P450 2E1-mediated toxicity studied in isolated periportal and perivenous hepatocytes. Biochemical Pharmacology, 45(6), 1251-1259. doi:10.1016/00062952(93)90277-4

Bessems, J. G. M., \& Vermeulen, N. P. E. (2001). Paracetamol (acetaminophen)-induced toxicity: Molecular and biochemical mechanisms, analogues and protective approaches. Critical Reviews in Toxicology, 31(1), 55-138. doi:10.1080/20014091111677

Boess, F., Kamber, M., Romer, S., Gasser, R., Muller, D., Albertini, S., et al. (2003). Gene expression in two hepatic cell lines, cultured primary hepatocytes, and liver slices compared to the in vivo liver gene expression in rats: Possible implications for toxicogenomics use of in vitro systems. Toxicological Sciences, 73(2), 386-402. doi:10.1093/toxsci/kfg064

Davidson, A. J., Ellis, M. J., \& Chaudhuri, J. B. (2010). A Theoretical Method to Improve and Optimize the Design of Bioartificial Livers. Biotechnology and Bioengineering, 106(6), 980-988. doi:10.1002/bit.22765

Davidson, A. J., Ellis, M. J., \& Chaudhuri, J. B. (2012). A theoretical approach to zonation in a bioartificial liver. Biotechnology and Bioengineering, 109(1), 234-243. doi:10.1002/bit.23279

Diaz Ochoa, J. G., Bucher, J., Pery, A. R. R., Zaldivar Comenges, J. M., Niklas, J., \& Mauch, K. (2012). A multiscale modeling framework for individualized, spatiotemporal prediction of drug effects and toxicological risk. Frontiers in pharmacology, 3, 204-204. doi:10.3389/fphar.2012.00204

Godoy, P., Hewitt, N., Albrecht, U., Andersen, M., Ansari, N., Bhattacharya, S., et al. (2013). Recent advances in $2 \mathrm{D}$ and $3 \mathrm{D}$ in vitro systems using primary hepatocytes, alternative hepatocyte sources and non-parenchymal liver cells and their use in investigating mechanisms of hepatotoxicity, cell signaling and ADME. Archives of Toxicology, 87(8), 1315-1530. doi:10.1007/s00204-013-1078-5

Hay, P. D., Veitch, A. R., \& Gaylor, J. D. S. (2001). Oxygen transfer in a convection-enhanced hollow fiber bioartificial liver. Artificial Organs, 25(2), 119-130. doi:10.1046/j.1525-1594.2001.025002119.x

Hinson, J. A., Roberts, D. W., \& James, L. P. (2010). Mechanisms of acetaminophen-induced liver necrosis. Handbook of experimental pharmacology(196), 369-405. doi:10.1007/978-3-642-00663-0_12

Jasmund, I., Langsch, A., Simmoteit, R., \& Bader, A. (2002). Cultivation of primary porcine hepatocytes in an OXY-HFB for use as a bioartificial liver device. Biotechnology Progress, 18(4), 839-846. doi:10.1021/bp025501y

Jungermann, K., \& Kietzmann, T. (2000). Oxygen: Modulator of metabolic zonation and disease of the liver. Hepatology, 31(2), 255-260. doi:10.1002/hep.510310201 
Katayama, S., Tateno, C., Asahara, T., \& Yoshizato, K. (2001). Size-dependent in vivo growth potential of adult rat hepatocytes. American Journal of Pathology, 158(1), 97-105. doi:10.1016/s00029440(10)63948-x

Kyffin, J. A., Sharma, P., Leedale, J., Colley, H. E., Murdoch, C., Mistry, P., et al. (2018). Impact of cell types and culture methods on the functionality of in vitro liver systems - A review of cell systems for hepatotoxicity assessment. Toxicology in Vitro 48, 262-275.

Lerche-Langrand, C., \& Toutain, H. J. (2000). Precision-cut liver slices: characteristics and use for in vitro pharmaco-toxicology. Toxicology, 153, 221-253.

Lu, H. F., Lim, W. S., Zhang, P. C., Chia, S. M., Yu, H., Mao, H. Q., et al. (2005). Galactosylated poly(vinylidene difluoride) hollow fiber bioreactor for hepatocyte culture. Tissue Engineering, 11(11-12), 16671677. doi:10.1089/ten.2005.11.1667

Michalopoulos, G., Sattler, G. L., \& Pitot, H. C. (1976). Maintenance of microsomal cytochromes B5 and P450 in primary cultures of parenchymal liver-cells on collagen membranes. Life Sciences, 18(10), 1139-1144. doi:10.1016/0024-3205(76)90149-1

Nantasanti, S., de Bruin, A., Rothuizen, J., Penning, L. C., \& Schotanus, B. A. (2016). Concise Review: Organoids Are a Powerful Tool for the Study of Liver Disease and Personalized Treatment Design in Humans and Animals. STEM CELLS Translational Medicine, 5(3), 325-330. doi:10.5966/sctm.20150152

Naritomi, Y., Terashita, S., Kagayama, A., \& Sugiyama, Y. (2003). Utility of Hepatocytes in Predicting Drug Metabolism: Comparison of Hepatic Intrinsic Clearance in Rats and Humans in Vivo and in Vitro. Drug Metabolism and Disposition, 31(5), 580.

Pang, K. S., Barker, F., Simard, A., Schwab, A. J., \& Goresky, C. A. (1995). Sulfation of acetaminophen by the perfused-rat-liver - the effect of red blood cell carriage. Hepatology, 22(1), 267-282. doi:10.1016/0270-9139(95)90381-x

Pery, A. R. R., Brochot, C., Zeman, F. A., Mombelli, E., Desmots, S., Pavan, M., et al. (2013). Prediction of dose-hepatotoxic response in humans based on toxicokinetic/toxicodynamic modeling with or without in vivo data: A case study with acetaminophen. Toxicology Letters, 220(1), 26-34. doi:10.1016/j.toxlet.2013.03.032

Plettenberg, S., Weiss, E. C., Lemor, R., \& Wehner, F. (2008). Subunits alpha, beta and gamma of the epithelial $\mathrm{Na}+$ channel $(\mathrm{ENaC})$ are functionally related to the hypertonicity-induced cation channel (HICC) in rat hepatocytes. Pflugers Archiv-European Journal of Physiology, 455(6), 1089-1095. doi:10.1007/s00424-007-0355-7

Prot, J. M., Briffaut, A.-S., Letourneur, F., Chafey, P., Merlier, F., Grandvalet, Y., et al. (2011). Integrated Proteomic and Transcriptomic Investigation of the Acetaminophen Toxicity in Liver Microfluidic Biochip. Plos One, 6(8). doi:10.1371/journal.pone.0021268

Prot, J. M., Maciel, L., Bricks, T., Merlier, F., Cotton, J., Paullier, P., et al. (2014). First pass intestinal and liver metabolism of paracetamol in a microfluidic platform coupled with a mathematical modeling as a means of evaluating ADME processes in humans. Biotechnology and Bioengineering, 111(10), 20272040. doi:doi:10.1002/bit.25232

Reddyhoff, D., Ward, J., Williams, D., Regan, S., \& Webb, S. (2015). Timescale analysis of a mathematical model of acetaminophen metabolism and toxicity. Journal of Theoretical Biology, 386, 132-146. doi:10.1016/j.jtbi.2015.08.021

Reith, D., Medlicott, N. J., Kumara De Silva, R., Yang, L., Hickling, J., \& Zacharias, M. (2009). Simultaneous modelling of the Michaelis-Menten kinetics of paracetamol sulphation and glucuronidation. Clinical and Experimental Pharmacology and Physiology, 36(1), 35-42. doi:10.1111/j.14401681.2008.05029.x

Remien, C. H., Adler, F. R., Waddoups, L., Box, T. D., \& Sussman, N. L. (2012). Mathematical modeling of liver injury and dysfunction after acetaminophen overdose: Early discrimination between survival and death. Hepatology, 56(2), 727-734. doi:10.1002/hep.25656

Ribeiro, A. C. F., Barros, M. C. F., Verissimo, L. M. P., Santos, C. I. A. V., Cabral, A. M. T. D. P. V., Gaspar, G. D., et al. (2012). Diffusion coefficients of paracetamol in aqueous solutions. Journal of Chemical Thermodynamics, 54, 97-99. doi:10.1016/j.jct.2012.03.014 
Rothbauer, M., Zirath, H., \& Ertl, P. (2018). Recent advances in microfluidic technologies for cell-to-cell interaction studies. J $R$ Soc Chemistry 18, 249-270.

Shipley, R. J., Davidson, A. J., Chan, K., Chaudhuri, J. B., Waters, S. L., \& Ellis, M. J. (2011). A Strategy to Determine Operating Parameters in Tissue Engineering Hollow Fiber Bioreactors. Biotechnology and Bioengineering, 108(6), 1450-1461. doi:10.1002/bit.23062

Shipley, R. J., Waters, S. L., \& Ellis, M. J. (2010). Definition and Validation of Operating Equations for Poly(Vinyl Alcohol)-Poly(Lactide-Co-Glycolide) Microfiltration Membrane-Scaffold Bioreactors. Biotechnology and Bioengineering, 107(2), 382-392. doi:10.1002/bit.22815

Siegers, C. P., Rozman, K., \& Klaassen, C. D. (1983). Biliary excretion and enterohepatic circulation of paracetamol in the rat. Xenobiotica, 13(10), 591-596.

Soldatow, V. Y., LeCluyse, E. L., Griffith, L. G., \& Rusyn, I. (2013). In vitro models for liver toxicity testing. Toxicol. Res., 2, 23-39.

Storm, M. P., Sorrell, I., Shipley, R., Regan, S., Luetchford, K. A., Sathish, J., et al. (2016). Hollow Fiber Bioreactors for In Vivo-like Mammalian Tissue Culture. J Vis Exp., 111.

Suzuki, T., Shinjo, S., Arai, T., Kanai, M., \& Goda, N. (2014). Hypoxia and fatty liver. World J Gastroenterol., 20, 15087-15097.

Tibbitt, M. W., \& Anseth, K. S. (2009). Hydrogels as extracellular matrix mimics for 3D cell culture. Biotechnol. Bioeng. , 103, 655-663.

Watari, N., Iwai, M., \& Kaneniwa, N. (1983). Pharmacokinetic study of the fate of acetaminophen and its conjugates in rats. Journal of Pharmacokinetics and Biopharmaceutics, 11(3), 245-272.

Williams, D. P., Shipley, R., Ellis, M. J., Webb, S., Ward, J., Gardner, I., et al. (2013). Novel in vitro and mathematical models for the prediction of chemical toxicity. Toxicology Research, 2(1), 40-59. doi:10.1039/c2tx20031g

Xia, L., Ng, S., Han, R., Tuo, X., Xiao, G., Leo, H. L., et al. (2009). Laminar-flow immediate-overlay hepatocyte sandwich perfusion system for drug hepatotoxicity testing. Biomaterials, 30(30), 5927-5936. doi:10.1016/j.biomaterials.2009.07.022 